\title{
Wound Botulism in a Drug User-A Case Report and a Review of Botulism
}

\author{
Leila Darki, Arash Jalali-Sohi, and Said R Beydoun \\ Neuromuscular Division, Department of Neurology, Keck School of Medicine, University of Southern California, LOS Angeles, CA, USA
}

DOI: https://doi.org/10.17925/USN.2020.16.2.116

\begin{abstract}
ntroduction: Wound botulism has been reported among injection drug users; however, diagnosis can be challenging, and initial misdiagnosis can delay appropriate treatment. Objective: To increase the clinical awareness of wound botulism and its symptoms to reduce misdiagnosis and improper treatment. Case study: A 43-year-old Caucasian male with a history of daily heroin use presented with acute onset descending weakness. Neurological examination showed bilateral ptosis, facial diplegia, and proximal weakness with diffuse hyporeflexia. Electrodiagnostic study demonstrated normal median and spinal accessory nerve compound muscle action potential amplitudes without an incremental response after a 10-second exercise. Median nerve repetitive nerve stimulation at $2 \mathrm{~Hz}$ revealed a decremental response at rest. Wound botulism was suspected despite lack of typical electrodiagnostic findings. The patient received botulism antitoxin, with partial improvement of his neurological symptoms. Mouse bioassay (MBA) with serum sample from the patient was negative; however, botulinum toxin type A was detected in serum via matrix-assisted laser desorption/ionization time-of-flight mass spectrometry. Conclusion: Despite the absence of classic electrodiagnostic findings of a presynaptic disorder and a negative MBA, clinicians should remain aware of wound botulism in particularly at-risk patients.
\end{abstract}

\section{Keywords}

Injection drug user, botulism,

MALDI-TOF, mouse bioassay, presynaptic, neuromuscular junction, SNARE complex

Disclosures: Said R Beydoun has received research/grant support from Argenx, Catalyst, Mallinkrodt, Ra Pharma, and UCB, and has served as a consultant/speaker for Akcea, Alexion, Alnylam, CSL, Mitsubishi Tanabe, and Takeda. Leila Darki and Arash Jalali-Sohi have no financial or non-financial relationships or activities to declare in relation to this article.

Review Process: Double-blind peer review.

Compliance with Ethics: Written informed consent was not obtained from the patient for publication of this case report due to loss of follow-up; no identifying information or images have been included.

Authorship: The named authors meet the Internationa Committee of Medical Journal Editors (ICMJE) criteria for authorship of this manuscript, take responsibility for the integrity of the work as a whole, and have given final approval for the version to be published.

Access: This article is freely accessible at touchNEUROLOGY.com (C) Touch Medical Media 2020

Received: April 29, 2020

Accepted: July 23, 2020

Published Online: December 23, 2020

Citation: US Neurology. 2020;16(2):116-9

Corresponding Author: Leila Darki,

Neuromuscular Division, Department of Neurology,

Keck Medicine of the University of Southern California,

1520 San Pablo Street, Suite 3000, Los Angeles,

CA 90033, USA.

E: leila.darki@med.usc.edu

LinkedIn: www.linkedin.com/in/leila-darki-725703a8

Support: No funding was received in

the publication of this article.
Botulism is an extremely rare paralytic illness caused by neurotoxins produced by the bacterium Clostridium botulinum. ${ }^{1}$ While much less common, botulism can result from infection by $\mathrm{C}$. butyricum and $C$. baratii as well. ' Found in soils and sediments worldwide, $C$. botulinum is a strictly anaerobic, Gram-positive, spore-forming bacterium that produces botulinum neurotoxins (BoNTs). ${ }^{2}$ There are currently eight known botulinum toxin serotypes, which are designated by the letters A through $\mathrm{H}^{3}{ }^{3}$ Of these eight known BoNTs, types A, B, E, and F are known to cause botulism in humans. ${ }^{4}$ BoNT is the most potent toxin known to humanity, with a lethal dose in humans of $1 \mu \mathrm{gg} / \mathrm{kg}$ if taken orally, 10-13 ng/kg if inhaled, and 1-2 ng/kg through intravenous or intramuscular routes. ${ }^{2}$ Foodborne botulism, wound botulism, infant botulism, and intestinal toxemia are the four recognized clinical syndromes caused by $\mathrm{C}$. botulinum infection. ${ }^{4}$ Iatrogenic botulism can occur through cosmetic or therapeutic injection. ${ }^{1}$ In 2017, 182 cases of laboratory-confirmed botulism occurred in the USA, according to the Centers for Disease Control and Prevention (CDC). ${ }^{5}$ Of these 182 cases, 141 were infant, 19 were foodborne, 19 were wound, two were iatrogenic, and one was suspected adult intestinal colonization. ${ }^{5}$

Foodborne botulism arises from the ingestion of food containing $C$. botulinum spores or bacteria that have been maintained in anaerobic conditions, allowing for germination and toxin production. ${ }^{6}$ Most frequently, the sources of foodborne botulism are mishandled homemade canned or raw fermented foods, as these provide the anaerobic conditions C. botulinum requires to thrive. ${ }^{4}$ Upon ingestion, BoNTs remain unharmed by gastric fluids due to associated accessory proteins that protect the toxins from proteolytic activity. ${ }^{6}$ These protective accessory proteins dissociate from the toxin in the alkaline environment of the small intestine. ${ }^{6}$ Once this dissociation occurs, the toxin is absorbed through the intestinal lining where it is carried in the blood to peripheral autonomic nerves and the nerve terminals of skeletal muscles. ${ }^{6}$

C. botulinum spores can contaminate wounded tissues, where they can germinate and produce BoNTs, causing wound botulism. ${ }^{4}$ As with foodborne botulism, the toxin is then carried to its target nerve cells via the circulatory system to exert its effects. ${ }^{6}$ Wound botulism is heavily associated with injection drug users (IDUs) who use "skin popping" to administer drugs subcutaneously. ${ }^{6}$ This condition is not exclusive to IDUs, as a small number of wound botulism cases have been attributed to the contamination of wounds caused by compound extremity fractures. ${ }^{4}$ 
In its active form, the BoNT protein consists of a $100 \mathrm{kDa}$ heavy chain and a $50 \mathrm{kDa}$ light chain.? The overall mechanism of action involves four key steps: cell binding, endocytosis, vesicular membrane translocation, and enzymatic cleavage of target proteins. ${ }^{7}$ First, BoNTs bind to gangliosides on the surface of neurons, and this complex then moves to bind to a receptor on the cell surface. ${ }^{2}$ This binding induces receptor-mediated endocytosis of the toxin, which is then thought to undergo a conformational change due to the acidic environment of the resulting endosome. ${ }^{2}$ The light chain is then translocated into the cytosol via a transmembrane protein-conducting channel formed by the heavy chain's insertion into the synaptic vesicular membrane. ${ }^{2}$ Through its $\mathrm{Zn}^{2+}$-dependent metalloprotease activity, the light chain cleaves proteins in the SNARE (soluble N-ethylmaleimide-sensitive fusion protein attachment protein receptors) complex that are essential to vesicle transportation and neurotransmitter release. ${ }^{2}$ Each BoNT serotype has unique hydrolytic cleavage sites within the SNARE complex. ${ }^{2}$ Toxin types $A$ and $E$ cleave unique peptide bonds on SNAP-25. ${ }^{6}$ BoNT serotypes $B, D, F$, and $G$ cleave synaptobrevin at distinctive single peptide bonds. ${ }^{6}$ BONT type $\mathrm{C}$ has cleavage sites on both syntaxin and SNAP-25. ${ }^{6}$ SNARE complexes maintain the presence of vesicles containing neurotransmitters at the presynaptic membrane. ${ }^{8}$ When an action potential reaches the axon terminal, the resulting $\mathrm{Ca}^{2+}$ influx causes these vesicles to fuse with the presynaptic membrane to release neurotransmitter into the synaptic cleft. ${ }^{8}$ When the SNARE complexes are cleaved by BONTs, there is a reduction in synaptic vesicle release. ${ }^{6}$ The resulting decrease in the endplate potential quantal content causes neuromuscular transmission failure. ${ }^{6}$

\section{Case report}

A 43-year-old Caucasian male was transferred to our hospital with a 5-day history of descending weakness. He had longstanding history of daily heroin use, including the injection of black tar heroin. Symptoms started with binocular diplopia, and over next 2 days he experienced slurring speech, swallowing difficulty, and weakness in his arms and legs. He denied any recent vaccination and prodromal respiratory or gastrointestinal symptoms.

On examination he was afebrile with a non-fluctuant abscess on the upper left extremity. Neurological examination showed bilateral ptosis and facial diplegia. His voice was nasal and dysarthric. He had weakness in proximal more than distal extremities with diffuse hyporeflexia. Sensory examination was normal. The admitting team considered the diagnosis of myasthenia gravis versus Guillain-Barré syndrome and administered a trial of intravenous immunoglobulin with a dosage of $2 \mathrm{~g} / \mathrm{kg}$, but his condition continued to worsen.

Electrodiagnostic study, performed 11 days after symptom onset, revealed normal median and spinal accessory nerve compound muscle action potential (CMAP) amplitudes and latency, but with unstable median CMAP response (amplitude variation on repeated single stimulation). There was no incremental response after a 10-second exercise. Median repetitive nerve stimulation (RNS) at $2 \mathrm{~Hz}$ revealed a $14.8 \%$ decremental response at rest. After a 30-second exercise, the decremental response decreased to 6.8\%. Spinal accessory nerve repetitive stimulation at $2 \mathrm{~Hz}$ was normal with no evidence of post-exercise facilitation or exhaustion. Electromyography study revealed increased insertional activity, positive sharp waves, and low amplitude polyphasic motor unit action potentials. Routine nerve conduction study was normal without evidence of demyelinating or axonal loss changes.
Based on the clinical suspicion of wound botulism, the patient was infused with a single dose (one vial) of BAT (botulism antitoxin heptavalent $[A, B, C, D, E, F, G]$-equine). An allergy test was not conducted prior to the administration of antitoxin. The abscess was derided and irrigated, and antibiotic treatment was initiated. The patient had partial improvement in neurological symptoms with no respiratory compromise, and then was transferred to an in-network facility. Mouse bioassay (MBA) was conducted using serum sample derived from the patient 13 days after symptom onset, but prior to BAT treatment. This assay yielded a negative result for botulism. A sample was also sent to the CDC, where matrix-assisted laser desorption/ionization time-of-flight mass spectrometry (MALDI-TOF MS) confirmed the presence of botulinum toxin type A.

\section{Discussion}

Wound botulism in chronic drug users is a well-known entity and has been described in the literature. ${ }^{9,10}$ Acute bulbar weakness with visual symptoms in patients with subcutaneous heroin abuse strongly suggests the possibility of wound botulism. High diagnostic suspicion combined with histology and in vitro electrophysiology confirmation of pre-synaptic failure, especially in seronegative cases, may significantly improve morbidity. ${ }^{10}$ However, patients with botulism are still frequently misdiagnosed because its symptoms mimic those of myasthenia gravis, Lambert-Eaton myasthenic syndrome, or Guillain-Barré syndrome. ${ }^{11}$ Since it is rare and mimics other conditions, botulism is not usually considered in the differential diagnosis. Specifically in patients who are IDUS, the average interval between the first medical consultation for symptoms of wound botulism and a physician first considering the condition is 3.1 days. ${ }^{12}$ Misdiagnosis, as occurred initially with our patient, further extends this diagnostic delay and frequently leads to patients receiving inappropriate care. ${ }^{13}$ This is detrimental to their healthcare outcomes because botulism antitoxin treatment is more effective the earlier it is administered, as it inactivates circulating BoNTs. ${ }^{13,14}$ This prevents further disease progression, but does not reverse neurological deficits that are already present. ${ }^{14}$ The receipt of inappropriate treatment only allows for the further progression of neurological symptoms..$^{13}$ It is important to note that patients receiving BAT may experience hypersensitivity reactions, allergic reactions, and other adverse effects. ${ }^{14}$ Symptoms of such reactions include anaphylaxis, urticaria, fever, chills, edema, and urinary retention. ${ }^{14}$ Prior to administration, it is not recommended that BAT sensitivity testing be carried out due to concerns regarding its accuracy and the delaying of treatment. ${ }^{14}$ Instead, patients should be closely monitored after BAT administration so that symptoms, should they arise, can be identified and treated. ${ }^{14}$

Symptoms of botulism include symmetric cranial nerve and bulbar palsies. ${ }^{4,11}$ Patients exhibiting these palsies may exhibit dysarthria, dysphagia, diplopia, ptosis, and facial muscle weakness., ${ }^{4,11}$ As the condition progresses, patients experience descending muscle weakness in the extremities accompanied by the loss of deep tendon reflexes. ${ }^{1}$ Descending paralysis can weaken the diaphragm and accessory respiratory muscles, causing patients to experience respiratory distress. ${ }^{1}$ Nausea, vomiting, and diarrhea may occur in patients with foodborne botulism; however, constipation is a hallmark symptom of infant botulism. ${ }^{4}$ Patients may also present with autonomic dysfunction, such as anhidrosis, reduced tear production, and decreased salivation. ${ }^{4,11}$ Patients maintain intact cognition, as BoNTs do not cross the blood-brain barrier. ${ }^{4}$ 
Electrodiagnostic studies are a useful tool for the diagnosis of botulism, but the findings of these studies may vary depending on the duration of infection. ${ }^{15,16}$ Electrodiagnostic studies of patients with botulism usually reveal decreased CMAP amplitudes with normal latencies and conduction velocities. ${ }^{15}$ Patients may also display a decremental response with low frequency RNS. ${ }^{15}$ With high rates of RNS or after 10 seconds of voluntary contraction, an incremental response in the CMAP amplitude can be identified. ${ }^{15}$ This finding, however, is usually present in early or mild cases. ${ }^{17}$ In severe botulism, the amount of acetylcholine release can drop severely below threshold. ${ }^{17}$ Facilitation from rapid RNS or brief exercise may not result in a threshold response, and no increment will occur in the CMAP amplitude. ${ }^{17}$ Thus, the lack of an incremental response to rapid RNS or brief exercise cannot completely exclude the diagnosis of botulism. ${ }^{17}$ Needle electromyography of botulism can display signs of active denervation, such as fibrillation potentials and positive sharp waves. ${ }^{17}$ Similar to other neuromuscular junction disorders, volitional activity can be normal, or may demonstrate polyphasic, short duration, low amplitude motor units resembling myopathic units. ${ }^{17}$ Depending on the severity, recruitment may be normal, early, or reduced. ${ }^{17}$ Reduced recruitment may occur if every muscle fiber of a motor unit is blocked by the botulinum toxin, effectively reducing the number of available motor units. ${ }^{17}$

Our patient's electrodiagnostic study displayed a decremental response with low frequency RNS at $2 \mathrm{~Hz}$; however, baseline CMAP amplitude was normal and did not demonstrate an incremental response after a 10-second exercise. CMAP response showed variability in amplitude with single stimuli, hinting to a presynaptic abnormality. Cherington reports that post-tetanic facilitation is seen more often in type B botulism than in type $A .{ }^{18}$ BoNT serotypes $A$ and $B$ have different molecular targets within the SNARE complex, which may account for the larger percentage of incremental responses in patients with type $B$ intoxication in comparison to those with type A intoxication. ${ }^{6}$

There are numerous methods that can be used to provide laboratory confirmation of botulism, but few have been validated for use in public health laboratories. ${ }^{19}$ Because of their relevance to this case report, only MBA and MALDI-TOF MS will be elaborated upon. MBA is an in vivo assay that serves as the standard method for detecting and confirming the presence of BoNTs in food samples, environmental samples, and clinical specimens. ${ }^{19}$ Samples for testing must be purified and diluted for use in this assay. ${ }^{19}$ Mice receive an intraperitoneal injection of the prepared sample, and are observed for symptoms of botulism. ${ }^{19} \mathrm{~A}$ cross-neutralization test is used to determine the BoNT serotype, whereby mice are injected with monovalent antibodies specific to each serotype. ${ }^{19}$ The mouse that receives the antitoxin that neutralizes the injected BoNT will show improvement, thus indicating the BoNT serotype. ${ }^{19}$ While MBA is the standard practice for confirmation of botulism, it does carry limitations. MBA is a costly and time-intensive method that requires skilled personnel and an animal care facility. ${ }^{19}$ Furthermore, full test completion can take between 1 and 4 days. ${ }^{19}$ This delay can be detrimental to treatment efforts, as antitoxin therapy is most effective when administered early in the disease course. ${ }^{13}$ As shown in this case, as well as with information from the CDC, this assay can frequently give false-negatives..$^{13}$ With a condition that is time-sensitive, false-negatives can damage patient outcomes by further delaying appropriate treatment.

MALDI-TOF MS can be 10-100 times more sensitive than MBA. ${ }^{20}$ MALDI-TOF MS can use intact bacteria or prepared protein extracts. ${ }^{21}$ A matrix, such as $\alpha$-cyano-4-hydroxycinnamic acid dissolved in 50\% acetonitrile and $2.5 \%$ trifluoroacetic acid, is used to aid in the desorption and ionization of bacterial analytes by protecting the sample from fragmentation upon bombardment with a laser. ${ }^{21}$ When the laser bombards the sample, matrix and bacterial molecules are desorbed and enter the gaseous state. ${ }^{21}$ Since the matrix absorbs most of the laser's energy, it becomes ionized. ${ }^{21}$ Through random collisions, the ionized matrix molecules transfer their charge to desorbed molecules from the bacterial sample. ${ }^{21}$ These now-ionized microbial molecules are accelerated into a time-of-flight mass analyzer based on their mass-to-charge ratio. ${ }^{21}$ Smaller analytes travel faster and reach the detector first, followed by progressively larger ions. ${ }^{21}$ This allows for the creation of a mass spectrum that indicates the number of ions of a particular mass impacting the detector with time. ${ }^{21}$ The generated mass spectrum serves as a unique "fingerprint" for the sample. For intact microbes, the mass spectrum can identify organisms based on peaks that are specific to particular genera, species, or strains. ${ }^{21}$ In the context of botulism, the unique cleavage sites by each serotype on their target proteins is used to determine toxin type. ${ }^{22}$ Each BoNT serotype cleaves at a specific site in the SNARE complex to produce products of unique molecular weights. ${ }^{22}$ By mixing a BoNT sample with proteins from the SNARE complex, the serotype can be determined by obtaining a mass spectrum of the cleavage products. ${ }^{22}$ MALDI-TOF MS can provide faster and more accurate results than MBA, but it is not widely available for public health use..$^{20}$

Knowing the patient was a daily IDU presenting with acute-onset symmetric cranial nerve and bulbar palsies and descending weakness, the decision was made to move forward with botulism antitoxin treatment despite the atypical electrodiagnostic findings. After the administration of botulism antitoxin, the patient showed partial improvement in his neurological symptoms. This reveals the importance of holistically reviewing a patient's case when determining diagnosis and treatment.

\section{Conclusion}

While exceptionally rare, it is important that healthcare providers maintain botulism in their differential diagnosis when patients present with acute-onset symmetric cranial nerve palsies, descending extremity muscle weakness, dysphagia, dysarthria, and areflexia. Electrodiagnostic studies may not display typical features, so such a finding should not immediately rule botulism out of the differential diagnosis. Furthermore, a negative result from an MBA should not be used to definitively rule out botulism because this assay carries with it, the limitation of false-negatives. It is important that healthcare providers are aware that IDUs are an at-risk population for wound botulism to reduce misdiagnosis. If possible, samples from patients with suspected botulism should also be sent for analysis by MALDI-TOF MS; however, results from this test may take 2-4 weeks because it is not widely available at this time..$^{20}$ If botulism is suspected, it is imperative that antitoxin treatment is initiated as soon as possible. $\square$ 
1. Chatham-Stephens KD, Fleck-Derderian SD, Johnson SD, et al. Clinical features of foodborne and wound botulism: a systematic review of the literature, 1932-2015. Clin Infect Dis. 2017;66(Suppl. 1):S11-6.

2. Kumar R, Dhaliwal H, Kukreja R, et al. The botulinum toxin as a therapeutic agent: molecular structure and mechanism of action in motor and sensory systems. Semin Neurol. 2016;36:10-9.

3. Barash JR, Arnon SS. A novel strain of clostridium botulinum that produces type $B$ and type $H$ botulinum toxins. IInfect Dis. 2013;209:183-91.

4. Carrillo-Marquez MA. Botulism. Pediatr Rev. 2016;37:183-92.

5. Centers for Disease Control and Prevention (CDC). National Botulism Surveillance Summary, 2017. Available at: www.cdc.gov/botulism/surv/2017/index.html (accessed August 4, 2020).

6. Maselli RA, Bakshi N. Botulism. Muscle Nerve. 2000;23: $1137-44$

7. Maselli RA. Pathogenesis of human botulism. Ann N Y Acad Sci. 1998;841:122-9.

8. Sudhof TC, Rothman JE. Membrane fusion: grappling with SNARE and SM proteins. Science. 2009;323:474-7.
9. Macdonald KL, Rutherford GW, Friedman SM, et al. Botulism and botulism-like illness in chronic drug abusers. Ann Intern Med. 1985;102:616-8.

10. Maselli RA, Ellis W, Mandler RN, et al. Cluster of wound botulism in California: clinical, electrophysiologic, and pathologic study. Muscle Nerve. 1997;20:1284-95.

11. Sobel J. Botulism. Clin Infect Dis. 2005;41:1167-73.

12. Werner SB, Passaro D, Mcgee J, et al. Wound botulism in California, 1951-1998: recent epidemic in heroin injectors. Clin Infect Dis. 2000;31:1018-24.

13. Centers for Disease Control and Prevention (CDC). Botulism in the United States, 1899-1996 - Handbook for Epidemiologists, Clinicians, and Laboratory Workers. 1998. Available at: https:// stacks.cdc.gov/view/cdc/6673 (accessed August 4, 2020).

14. Yu PA, Lin NH, Mahon BE, et al. Safety and improved clinical outcomes in patients treated with new equine-derived heptavalent botulinum antitoxin. Clin Infect Dis. 2017;66(Suppl. 1):S57-64.

15. Chang VH, Robinson LR. Serum positive botulism with neuropathic features. Arch Phys Med Rehabil. 2000;81:122-6.

16. Lispi L, Leonardi L, Petrucci A. Longitudinal neurophysiological assessment of intramuscular type-A botulin toxin in healthy humans. Neurol Sci. 2018;39:329-32.

17. Preston DC, Shapiro BE, Neuromuscular Junction Disorders. In: Preston DC, Shapiro BE (eds). Electromyography and Neuromuscular Disorders Clinical-Electrophysiologic Correlations. London: Elsevier Saunders, 2013;529-48.

18. Cherington $\mathrm{M}$. Electrophysiologic methods as an aid in diagnosis of botulism: a review. Muscle Nerve Suppl. 1982;5:S28-9.

19. Thirunavukkarasu N, Johnson E, Pillai S, et al. Botulinum neurotoxin detection methods for public health response and surveillance. Front Bioeng Biotechnol. 2018;6:20.

20. Halai UA, Terashita D, Kim M, et al. Notes from the field: intestinal colonization and possible iatrogenic botulism in mouse bioassaynegative serum specimens - Los Angeles County, California, November 2017. MMWR Morb Mortal WKIJ Rep. 2018:67: $1221-2$.

21. Patel R. MALDI-TOF MS for the diagnosis of infectious diseases. Clin Chem. 2014;61:100-11.

22. Barr JR, Moura H, Boyer AE, et al. Botulinum neurotoxin detection and differentiation by mass spectrometry. Emerg Infect Dis. 2005;11:1578-83. 